\title{
The concentration-dependent aggregation of Ag NPs induced by cystine
}

\author{
Afshinnia K, Gibson I, Merrifield R and Baalousha $\mathbf{M}^{\star}$ \\ Center for Environmental Nanoscience and Risk, Department of Environmental Health \\ Sciences, Arnold School of Public Health, University South Carolina, Columbia, South \\ Carolina 29208 \\ Corresponding author:* mbaalous@mailbox.sc.edu.
}

\begin{abstract}
Cystine is widely used in cell culture media. Cysteine, the reduced form of cystine, is widely used to scavenge dissolved $\mathrm{Ag}$ in eco-toxicological studies to differentiate dissolved vs. nanoparticle uptake and toxicity. However, little is known about the impact of cysteine and cystine on the aggregation behavior of $\mathrm{Ag} \mathrm{NPs}$, in particular as a function of Ag NP concentration. Herein, we investigate how cystine (0-300 $\mu \mathrm{M})$ affects the stability of citrate-, polyvinylpyrrolidone-, and polyethylene glycol- coated silver nanoparticles (cit-Ag NPs, PVP-Ag NPs and PEG-Ag NPs, respectively) with and without Suwannee River fulvic acid (SRFA) as a function of Ag NPs concentration using UV-vis spectroscopy at environmentally and ecotoxicologically relevant Ag NP concentrations (ca. 125-1000 $\mathrm{Mg} \mathrm{L}^{-1}$ ). The results demonstrate, for the first time, the concentration-dependent aggregation of cit-Ag NPs in the presence of cystine with a shift in the critical coagulation concentration (CCC) to lower cystine concentrations at lower cit-Ag NP concentrations. At the highest cit-Ag NP concentration (1000 $\mu \mathrm{L}^{-1}$ ), reaction limited aggregation was only observed and no CCC was measured. SRFA slowed the aggregation of cit-Ag NPs by cystine and aggregation occurred in reaction limited aggregation (RLA) regime only. No CCC value was measured in the presence of SRFA. Cystine replaces citrate, PVP and PEG coatings, resulting in aggregation of both electrostatically and sterically stabilized Ag NPs. These findings are important in understanding the factors determining the behavior of Ag NPs in cell culture media. Also due to the similarity between cystine and cysteine, these results are important in understanding the uptake and toxicity of $\mathrm{Ag}$ NPs vs. Ag ions, and suggest that the reduction of the toxicity of Ag NPs in the presence of cysteine could be due to a combined effect of scavenging $\mathrm{Ag}^{+}$ions and Ag NP aggregation in the presence of cysteine.
\end{abstract}




\section{Introduction}

The rapid development of nanotechnology and concern regarding the potential environmental and human health implication of nanoparticles (NPs) has spurred extensive interest in understanding the behavior, fate and effects of NPs ${ }^{1-3}$. Silver NPs (Ag NPs) are among the most widely used NPs in consumer products as antimicrobial agent ${ }^{4}$. Several studies have suggested that Ag NPs can be released from nano-enabled products and find their way to the environment, resulting in environmental and human exposure to $\mathrm{Ag} \mathrm{NPs}^{5,6}$.

Considerable research has been conducted to better understand the effect of Ag NPs on a wide range of organisms and cell lines ${ }^{7}$, with a particular attention given to differentiate the NP from dissolved metal effects due to the known solubility of $\mathrm{Ag} \mathrm{NPs}{ }^{8-11}$. Several studies have reported mixed results; some show particle-specific toxicity ${ }^{8,12-14}$, others predominantly relate the effect to free $\mathrm{Ag}^{+}$ions ${ }^{15,16}$, while other studies suggested combined effect of $\mathrm{Ag}$ NPs and free $\mathrm{Ag}^{+}$ions ${ }^{17,18}$. These controversies could be partially due to the lack of understanding of NP behavior in the ecotoxicological media $^{7}$, in particular at eco-toxicologically relevant concentrations $^{19,20}$.

To differentiate $\mathrm{Ag}^{+}$from Ag NPs effect, binding ligands (e.g. $\mathrm{Cl}^{-}$and cysteine) are typically used to complex the released silver ions from $\mathrm{Ag} \mathrm{NPs}^{8,18}$, with little understanding of the impact of the ligands (e.g. cysteine) on the behavior of Ag NPs in the test medium. Several studies have reported reduced toxicity of Ag NP suspension in the presence of cysteine and attributed this reduction solely to the complexation of free $\mathrm{Ag}^{+}$ions by cysteine ${ }^{8,18}$, without considering the potential destabilization of Ag NPs in the presence of cysteine. Therefore, understanding the interaction between Ag NPs and cysteine is an important issue to underpin the assessment of dissolved silver and Ag NP uptake and toxicity in aquatic organisms. Additionally cystine, the oxidized form of cysteine, is widely used in cell culture media (http://www.sigmaaldrich.com/lifescience/cell-culture/learning-center/media-expert/cysteine.html). Cysteine and cystine are essential amino acids in cell culture media that must be supplied from an exogenous source.

On the other hand, the fate, transport and effects of Ag NPs in the environment depends on their interaction with naturally ubiquitous organic ligands such as thiols (cysteine), disulfide (cystine) and natural organic matter (NOM; fulvic and humic acids), which may chemically, or physically sorb onto NP surface and modify Ag NPs surface properties and colloidal stability ${ }^{21,22}$. For example hydrosulfide groups in NOM can form strong covalent bonds with Ag NPs surface and replace the original surface coating such as polyvinylpyrrolidone $(P V P)^{23,24}$, and thus reduce PVP-Ag NPs stability by increasing ionic strength ${ }^{25}$. Aggregation of NPs (e.g. Ag NPs and Au NPs) can lead to a reduction in NP toxicity ${ }^{26,27}$. 
Few studies have investigated the effect of cysteine on the stability of $\mathrm{Ag} \mathrm{NPs}^{22}$ and their fate and transport in porous media ${ }^{21}$, typically at high Ag NP concentrations (ca. $>1 \mathrm{mg} \mathrm{L}$ $\left.{ }^{1}\right)^{7,21,22}$, and observed slight aggregation of cit- and PVP- Ag NPs over a period of 48 hours $^{25}$. However, there are currently no studies on the impact of cystine on the behavior of Ag NPs, in particular at low NP concentrations.

The aim of this study is therefore to investigate the stability of citrate-, PVP- and PEGcoated Ag NPs following their interaction with cystine. In particular, we investigated the impact of cystine on cit-Ag NP aggregation kinetics at environmentally and eco-toxicologically relevant Ag NP concentrations (125-1000 $\left.\mathrm{g} \mathrm{L}^{-1}\right)$, and the impact of SRFA on the interaction between citAg NPs and cystine. We also investigated the aggregation of citrate, PVP and PEG-Ag NPs up to 24 hours post interaction with different concentrations of cystine. Cystine (disulfide form of cysteine) and Suwannee River fulvic acid (SRFA) were chosen to represent two distinct types of NOM. Cystine and cysteine represent a class of small molecular weight metal-complexing ligands with a thiol and disulfide groups and are commonly present in surface waters at low concentrations (nM to $\mu \mathrm{M}$ range $)^{25,28}$. Cysteine is also widely used in Ag NP toxicity experiments to differentiate the toxicity of NP vs. $\mathrm{Ag}^{+}$ions. Cystine rather than cysteine was used in this study for the following reasons 1) the weaker affinity of the disulfide group to $\mathrm{Ag}^{+}$, thus minimizing the impact on Ag NP dissolution and enabling the use of UV-vis to study the aggregation behavior of Ag NPs, 2) cysteine is highly reactive and readily oxidizes to cystine under oxic conditions ${ }^{29,30}$, and 3) Cystine is the predominant form of in plasma, extracellular body fluids and cell culture media ${ }^{31}$. SRFA represent a class of less-defined natural macromolecules, which usually stabilize NPs ${ }^{19,32}$.

\section{Materials and Methods}

\section{Chemicals}

Silver nitrate (ACS grade, $99.9^{+} \%$ ) and Sodium borahydrate (98\%) were purchased from Alfa Aesar (Ward Hill, MA, USA). Trisodium citrate (Lab grade) was purchased from Fisher Scientific (Pittsburgh, PA, USA). Cystine (L-cystine $\geq 98 \%$ TLC) was purchased from Sigma Aldrich (St Louis, MO, USA). SRFA was purchased from the International Humic Substances Society (St Paul, MN, USA). All chemicals were used without further purification.

\section{Synthesis and characterization of silver nanoparticles}

Citrate-coated silver NPs (cit-Ag NPs) were synthesized and characterized as described in previous publications ${ }^{33,34}$. Briefly, cit-Ag NPs (cit-Ag NPs) were prepared by the reduction of 
silver nitrate in trisodium citrate. A $100 \mathrm{ml}$ of $0.31 \mathrm{mM}$ trisodium citrate, $100 \mathrm{ml}$ of $0.25 \mathrm{mM}$ silver nitrate and $10 \mathrm{mM}$ of sodium borohydride solutions were prepared in high purity water and kept at $4{ }^{\circ} \mathrm{C}$ in the dark for $30 \mathrm{~min}$. The silver nitrate and trisodium citrate solutions were mixed together in a conical flask and vigorously stirred. Subsequently, $6 \mathrm{ml}$ of the reducing agent, sodium borohydride $\left(\mathrm{NaBH}_{4}\right)$, was added in one batch. After $10 \mathrm{~min}$ of stirring, the solution was heated slowly to boiling and heated for a further $90 \mathrm{~min}$, left overnight and cooled $\left(4{ }^{\circ} \mathrm{C}\right.$, in the dark). Ag NPs were then cleaned, to remove the excess reagents before use, by ultrafiltration (Amicon, $1 \mathrm{kDa}$ regenerated cellulose membrane, Millipore) using a diafiltration method to prevent NP aggregation and drying. Ag NPs were re-dispersed in $0.31 \mathrm{mM}$ trisodium citrate solution to avoid further growth; this process was repeated at least three times. The concentration of Ag NPs was measured by inductively coupled plasma-optical emission spectroscopy and was about $10.0 \pm 0.3 \mathrm{mg} \mathrm{L}^{-1}$.

PVP- and PEG- Ag NPs were prepared by ligand exchange approach using citratecoated Ag NPs as precursor as described elsewhere ${ }^{35}$. Aliquots of cit-Ag NPs were converted into PEG- and PVP- stabilized Ag NPs by adding 8 molecules of PVP, or 4 molecules of PEG per $\mathrm{nm}^{2}$ surface area of Cit-Ag NPs under vigorous stirring (e.g. $700 \mathrm{rpm}$ ) for at least 1 hour and then cooled overnight at $4^{\circ} \mathrm{C}$.

The size and electrophoretic mobility (EPM) was measured by dynamic light scattering and laser Doppler electrophoresis, respectively using a Malvern Zetasizer NanoZS Instrument (Malvern, USA). The Malvern Zeta potential transfer standard consisting of Latex dispersed in $\mathrm{pH} 9.2$ buffer and has an EPM value of $-42 \mathrm{mV}$ (DTS1235, Malvern Instrument Limited) was used to verify the performance of the instrument and the zeta potential cell throughout the experiments.

The optical absorbance spectrum of Ag NPs were collected by a Uv-vis spectrometer (UV-2600 Shimadzu, Santa Clara, CA, USA) using a $100 \mathrm{~mm}$ path length cuvette, which allowed analysis of Ag NPs at relatively low concentrations (125 to $\left.1000 \mu \mathrm{g} \mathrm{L}^{-1}\right)$. Uv-vis spectra of cit-Ag NPs suspensions were collected over wavelengths $(\lambda)$ 200-900 nm.

\section{Solution chemistry}

The aggregation behavior of cit-Ag NPs was investigated by Uv-vis spectroscopy in suspensions containing variable concentration of Ag NPs (125 to $1000 \mu \mathrm{L} \mathrm{L}^{-1} ; 1.16-9.27 \mu \mathrm{M} \mathrm{Ag}$ ) and cystine ( 0 to $72 \mathrm{mg} \mathrm{L}^{-1} ; 0-300 \mu \mathrm{M}$ ), in the presence and absence of Suwannee River Fulvic acid (SRFA, $1 \mathrm{mg} \mathrm{L}^{-1}$ ). No buffer was used to avoid interferences from ions in the buffer solution. For instance, phosphate anions (a component of the commonly used phosphate 
buffers) have been shown to induce the aggregation of hematite particles ${ }^{36}$ and titanium dioxide $\mathrm{NPs}^{37}$. The $\mathrm{pH}$ was measured throughout the experiment and was in the range 5.5-5.7.

A stock suspension of SRFA was prepared by dissolving $2 \mathrm{mg}$ in $10 \mathrm{ml}$ ultrahigh purity water (UHPW), and the $\mathrm{pH}$ was adjusted by adding aliquots $\mathrm{NaOH}$ to $\mathrm{pH}$ 7.0. The suspension was then filtered $(450 \mathrm{~nm})$ to remove any aggregated SRFA molecules. A stock suspension of $333 \mu \mathrm{M}\left(80 \mathrm{mg} \mathrm{L}^{-1}\right)$ cystine was prepared by dissolving $8 \mathrm{mg}$ of cystine in $100 \mathrm{ml}$ of UHPW. The cystine stock suspension was prepared daily before performing the aggregation experiments to avoid losses of cystine by degradation ${ }^{25}$.

For all experiments performed in the absence of SRFA, an aliquot $(125,250,500$, and $1000 \mu \mathrm{L}$ ) of Ag NPs stock suspension was diluted in UHPW and mixed with different aliquots of cystine to achieve a final volume of $10 \mathrm{~mL}$, and thus NP concentrations of 125, 250, 500 and $1000 \mathrm{~g} \mathrm{~L} \mathrm{~L}^{-1}$. For all experiments performed in the presence of SRFA, an aliquot of 2 and $4 \mathrm{ml}$ of Ag NPs stock suspension was mixed with 0.8 and $0.4 \mathrm{ml}$ of SRFA stock suspension and topped with UHPW to achieve a final volume of $8 \mathrm{~mL}$, which resulted in a mixture of 20 and $10 \mathrm{mg} \mathrm{L}^{-1}$ SRFA and concentrations of Ag NPs of 2.5 and $5.0 \mathrm{mg} \mathrm{L}^{-1}$. The mixtures were then left for $24 \mathrm{~h}$ to reach equilibrium before performing any aggregation kinetic experiments. Then 0.5 and $1 \mathrm{ml}$ of the mixtures were mixed with cystine and topped with UHPW to $10 \mathrm{~mL}$ to achieve a final concentration of Ag NPs of 125 and $500 \mu \mathrm{g} \mathrm{L}^{-1}$, SRFA of $1 \mathrm{mg} \mathrm{L}^{-1}$, and cystine $(0-266 \mu \mathrm{M})$.

\section{Aggregation kinetics}

To quantitatively measure the aggregation kinetics of cit-Ag NPs by Uv-vis, the concentration of individual cit-Ag NPs was monitored over time by performing a time scan of the Uv-vis absorbance at $\lambda=394 \mathrm{~nm}$, corresponding to the specific plasmon resonance peak for the individual Ag NPs (Figure 1a). The changes in the UV-vis absorbance $\lambda=394 \mathrm{~nm}$ can be attributed to NP aggregation or dissolution ${ }^{32,38}$. Analysis of dissolved Ag concentration by ICPMS following centrifugal ultrafiltration at $3 \mathrm{kD}$ over 24 hours, well beyond the time required to detemine NP aggregation kinetics, demonstrated that the differences in cit-Ag NP dissolution in the presence or absence of cystine was not significant (Figure S1). Therefore, the changes in the UV-vis absorbance $\lambda=394 \mathrm{~nm}$ can be attributed to aggregation of primary Ag NPs. The aggregation rate constant is proportional to rate of change of the Uv-vis at $\lambda=394 \mathrm{~nm}\left(\mathrm{da}_{\lambda=394} / \mathrm{d}_{\mathrm{t}}\right.$, a refers to Uv-vis absorption, Eq.1) during the early stage aggregation ${ }^{32,39}$; that is slope of the loss in the UV-vis absorbance at $\lambda=394$ within the first 30 seconds after mixing Ag NPs with cystine, which was determined by fitting a linear correlation function. 


\section{Results and Discussion}

\section{Properties of the synthesized Ag NPs}

According to the cumulant analysis, the $z-d_{h}$ and polydispersity index of cit-Ag NPs in stock suspension are $16.6 \pm 0.2 \mathrm{~nm}, 0.2 \pm 0.1$, respectively. Those of PEG-Ag NPs were 19.4 \pm 0.3 , and $0.29 \pm 0.003$ and those of PVP-Ag NPs were $21.6 \pm 0.3 \mathrm{~nm}$ and $0.27 \pm 0.005$. The zeta 
potential of cit-Ag NPs, PEG-Ag NPs and PVP-Ag NPs was $-46.5 \pm 4.5 \mathrm{mV},-28.5 \pm 1.4 \mathrm{mV}$, and $23.6 \pm 1.1 \mathrm{mV}$, respectively. These measurements suggest that cit-, PEG- and PVP-Ag NPs are all well dispersed. The Uv-vis spectra of the cit-Ag NPs, PEG-Ag NPs and PVP-Ag NPs in UHPW water shows a single peak centered at 394 nm, 394 nm, and $395 \mathrm{~nm}$, respectively. Full characterization of the synthesized cit-Ag NPs are provided elsewhere ${ }^{32-34}$.

\section{Cystine induced aggregation of cit-Ag NPs}

The Uv-vis spectra of cit-Ag NPs, after 10 minutes of mixing with different concentrations of cystine (Figure 1a-d) show a broadening of the Uv-vis absorbance peak (centered on 394 $\mathrm{nm}$ ) of as-synthesized cit-Ag NPs at low cystine concentrations (ca. $20 \mu \mathrm{M}$ ). At higher concentrations of cystine (ca. 33-83 $\mu \mathrm{M}$ ), the Uv-vis spectra show the formation of a second peak centered on higher wavelengths in the visible and near infrared region (NIR) of the spectrum in the range of $500-650 \mathrm{~nm}$. The increase in cystine concentration results in the decrease in the Uv-vis absorbance at $394 \mathrm{~nm}$ and the increase of the second peak absorbance at $550-560 \mathrm{~nm}$ as a consequence of NP interactions, which indicates the loss of primary cit-Ag NPs by aggregation as observed elsewhere ${ }^{32}$. Furthermore, at the same concentration of cystine (ca. 33 and $67 \mu \mathrm{M}$, Figure S2), the second peak is shifted to higher wavelengths at lower NP concentrations compared to higher NP concentrations. The increased width of the second peak at lower NP concentrations is likely to be due to the formation of larger Ag NP aggregates $^{32}$, which can be attributed to the higher decrease in Ag NP surface charge with the decrease in cit-Ag NPs concentration (Figure 2a) at the same concentration of cystine (discussed below). Under such conditions, more cystine molecules are available for interaction with the Ag surface atoms at lower Ag NP concentrations.

The UV absorbance at $\lambda_{394}$ follows a first order decrease at low cystine concentrations and a more rapid, higher order decrease at high cystine concentrations (Figure 2a-c). At the same concentration of cystine (Figure S4), the relative loss in the UV-vis absorbance increase with the decrease in NP concentration, indicating higher removal of primary NPs by more aggregation at lower Ag NPs concentrations. The attachment efficiencies of cit-Ag NPs calculated from the loss of the Uv-vis at $\lambda_{394}$ are plotted in Figure 3a, which generally shows RLA and DLA regimes and suggests that the aggregation kinetic of cit-Ag NPs in the presence of cystine are controlled by electrostatic interactions between the cystine coated AgNPs (see discussion below). The increased cystine concentration results in a decrease in the surface charge of the cit-Ag NPs (Figure 2a). In the RLA regime, the cit-Ag NPs are kinetically stabilized by an electrostatic barrier resulting from the double layer repulsion and the van der 
Waals attractions forces. In the DLA, the surface charge of the cit-Ag NPs is sufficiently reduced by the cystine and the energy barrier is no longer sufficient to kinetically stabilize the particles, and therefore, aggregation process is only driven by diffusion. It was not possible to calculate the attachment efficiency at cit-Ag NP concentration of $1000 \mathrm{~g} \mathrm{~L} \mathrm{~L}^{-1} \mathrm{Ag}$ as the concentration of cystine, within its limit of solubility, was not sufficient to induce DLA and thus it was not possible to calculate the fast aggregation rate.

At the same cystine concentration and under RLA regime (Figure 3a), the attachment efficiency decreases with the increase in Ag NP concentration. This behavior can be attributed to the higher abundance of cystine per surface atom of Ag NPs at lower NP concentrations, resulting in higher coverage of the NP with cystine and therefore higher reduction in Ag NP surface charge. The CCC as a function of Ag NP concentration increases with the increase in NP concentration (Figure $\mathbf{3 b}$ ), which can be attributed again to the fact that cystine is more efficient in aggregating cit-Ag NPs at lower NP concentrations. This is the first time that cystineinduced concentration-dependent aggregation of cit-Ag NPs is demonstrated.

\section{Effect of cystine on cit-Ag NPs Surface charge}

Cit-Ag NPs are negatively charged (zeta potential of $-46.5 \pm 4.5 \mathrm{mV}$ ). Figure $2 \mathrm{a}$ shows the zeta potential of Ag NPs (125-1000 $\left.\mathrm{g} \mathrm{L}^{-1}\right)$ as a function of cystine concentration, and shows that, for a fixed NP concentration, the magnitude of zeta potential decrease with the increase in cystine concentration. For a fixed cystine concentration, the magnitude of zeta potential decrease with the decrease in NP concentration. These observations are in agreement with the increased aggregation of Ag NPs at higher cystine concentrations and lower NP concentrations.

High uncertainty was observed in the measured zeta potential at low NP concentrations as laser doppler electrophoresis requires high NP concentrations, in the range of several $\mathrm{mg} \mathrm{L}^{-1}$ ${ }^{41}$. Therefore, to further confirm the effect of cystine on the surface charge of cit-Ag NPs, a second set of experiments were performed at higher NP concentrations ( 1 to $3.5 \mathrm{mg} \mathrm{L}^{-1}$ ) and at fixed cystine concentration (225 $\mu \mathrm{M}$; Figure $2 \mathbf{b}$ ). Again, the results demonstrate that the magnitude of zeta potential of cit-Ag NPs decreased to lower values with the decrease in NP concentration (Figure 2b). Thus, cystine is more efficient in reducing the surface charge of citAg NPs at lower NP concentrations, which can be attributed to the higher cystine to surface $\mathrm{Ag}$ atom ratio at lower NP concentrations and thus the higher coverage of Ag NPs by cystine molecules.

The cystine-induced aggregation and surface charge alteration of cit-Ag NPs can be explained by the chemical interaction of the surface Ag atoms with the disulfide group in the 
cystine molecule and the zwitterion-type (Zwitterion is a neutral molecule with a positive and a negative electrical charge, though multiple positive and negative charges can be present) surface charge behavior of cystine molecules. It has been demonstrated that similar to the thiol group in cysteine, disulfide groups in cystine can sorb on the surface of $\mathrm{AgNPs}^{42,43}$. Additionally, Ag NPs have been shown to induce S-S cleavage in cystine at low concentrations, but can keep intact S-S bonds bond at a concentration threshold of about $200 \mu \mathrm{M}^{42,44,45}$, which may explain the similarity in the effect of cystine and cysteine in inducing the aggregation of silver NPs.

After cystine adsorption on the Ag NPs, the cystine molecule has still two functional groups (carboxylic and amine) to form bonds between particles. The $p K a$ value of the carboxylic and amine groups are 1.96 and 8.18 , respectively ${ }^{46}$ and the isoelectric pH of cystine is 5.07 . The $\mathrm{pH}$ of the Ag NPs-cystine mixture was about 5.5-5.7, thus cystine has a negative net charge at the experimental conditions. At this $\mathrm{pH}$, the amine functional groups of cystine are protonated (positively charged, $\mathrm{NH}^{3+}$ ), whereas the carboxylic groups are deprotonated (negatively charged, $\left.\mathrm{COO}^{-}\right)$. Thus, the positively charged amino group in the cystine $\left(-\mathrm{NH}^{3+}\right)$ on the surface of one NP interacts with the negative charge on the surface of other Ag NP (carboxylic group, $\mathrm{COO}^{-}$) through electrostatic attraction ${ }^{47,48}$, and cause aggregation ${ }^{46}$. Hence, the cystine molecule constitutes a bridge among Ag NPs, thus inducing Ag NPs aggregation.

The concentration-dependent aggregation of Ag NPs in the presence of cystine is due to the ratio between $\mathrm{Ag}$ and cystine, and thus the surface coverage of Ag NPs by cystine. At lower Ag NP concentration, more cystine molecules are available to react with the surface of Ag NPs, resulting in a higher surface coverage of Ag NPs by cystine, reduction of surface charge of $\mathrm{Ag}$ NPs, and increased aggregation of Ag NPs. This is further supported by the shift to higher wavelengths (approximately $10 \mathrm{~nm}$ ) in the Plasmon resonance of Ag NPs at lower NP concentrations in the presence of cystine (Figure 2c), indicating increased interaction with cystine at low NP concentrations.

\section{Aggregation impacted by fulvic acid}

The UV-vis spectra of cit-Ag NPs 10 minutes post interaction with cystine in the presence of SRFA show little to no change in the spectra in term of peak broadening or absorbance loss at $394 \mathrm{~nm}$ (Figure S5a and b), suggesting that SRFA prevents (or reduce) cystine induced aggregation of cit-Ag NPs. The rate of the decay $\lambda_{394}$ is generally slower and decays to higher absorbance in the presence of SRFA ( 0.88, Figure S5a and $\mathbf{b})$ compared to the absence of SRFA ( 0.6, Figure $2 \mathbf{a}$ and $\mathbf{c})$, presumably due to the reduced aggregation of cit-Ag NPs in the presence of SRFA. It was not possible to calculate the attachment efficiency in 
the presence of SRFA because aggregation of cit-Ag NPs did not reach the fast aggregation regime (DLA) within the limit of cystine solubility in water $(333 \mu \mathrm{M})$. Nonetheless, the rate loss of UV-vis ${ }_{394}$ increases slightly with the increase in cystine concentration, but does not reach the fast aggregation rate (Figure 4a).

It is well known that SRFA forms a surface coating on cit-Ag NPs and increase the surface charge and provide steric stabilization ${ }^{19,49-51}$ to cit-Ag NPs. Therefore, the sorption of SRFA molecules on cit-Ag NPs increases electrosteric stabilization of cit-Ag NPs, but they do not completely inhibit surface charge screening and NP aggregation by electrolytes ${ }^{32,52}$. It is worth noting that, in the presence of SRFA, the surface charge of cit-Ag NPs did not change with the increase in cystine concentrations (Figure 4b), which can be attributed to the lack of interaction between cystine and the surface of Ag NPs. Similarly to the case of without SRFA, another set of experiment was conducted to measure the zeta potential of Ag NPs 24 hours post interaction with cystine in the presence of SRFA at higher Ag NP concentrations (1.0-3.5 $\mathrm{mg} \mathrm{L}^{-1} \mathrm{Ag}$ ) to avoid any uncertainty in the measured zeta potential at low NP concentrations (Figure 4c). The results suggest that the magnitude of the zeta potential decreases with the decrease in NP concentration at constant cystine concentration $(225 \mu \mathrm{M})$, indicating the increased efficiency of surface charge alteration at lower NP concentrations due to the increased probability of surface Ag atoms-cystine interactions. Nonetheless, the magnitude of the zeta potential in the presence of SRFA (ca. -28 to $-44 \mathrm{mV}$ ) remains higher compared with that in the absence of SRFA (ca. -17 to $-24 \mathrm{mV}$ ), indicating that SRFA form a surface coating on Ag NPs, enhance their surface charge and thus their stability in the presence of cystine. The Uv-vis of Ag NPs spectra 24 hours post interaction with cystine in the presence of SRFA shows no shift in the first peak position with the change in NP concentrations (Figure 4d) confirming that SRFA limits the interaction of cystine with surface of Ag NPs. Furthermore, Figure 4d shows the formation of a second peak at higher wavelengths. For the same concentration of $\mathrm{Ag}$ NPs, the absorbance of the second peak is lower in the presence of SRFA (Figure 4d) compared with that in the absence of SRFA (Figure 2c), again confirming the role of SRFA in reducing the cystine-induced aggregation of Ag NPs.

\section{Aggregation impacted by NP surface coating}

It is well known that surface coatings have a significant impact on NP stability and aggregation. Whereas citrate and PEG stabilize Ag NPs via electrostatic repulsion, PVP stabilizes Ag NPs via steric stabilization mechanism ${ }^{32,35}$. Therefore, citrate and PEG coated Ag NPs are susceptible to aggregation by charge screening by electrolytes, whereas PVP coated 
Ag NPs are generally stable at a wide range of ionic strengths and $\mathrm{pHs}^{32,35}$. Here, we investigate the impact of cystine on the stability of PEG- and PVP-Ag NPs at ecotoxicologically relevant and near environmentally relevant Ag NP concentrations.

The UV-vis absorbance of Cit-, PEG- and PVP- coated Ag NPs $\left(225 \mu \mathrm{g} \mathrm{L}^{-1}\right)$ immediately after addition of different concentrations of cystine (7-133 $\mu$ ) are presented in Figure 5a, $\mathbf{d}$ and $\mathbf{f}$, together with those after 6 hours (Figure $\mathbf{5 b}$, e and $\mathbf{h}$ ) and 24 hours (Figure $\mathbf{5 c}$, $\mathbf{f}$ and $\mathbf{i}$ ). All spectra show two peaks, the first centered on 394 to $402 \mathrm{~nm}, 393-400 \mathrm{~nm}$ and 393-412.5 nm immediately after addition of cystine for citrate-, PVP- and PEG coated Ag NPs, respectively. The position of the first peak shifts to higher wavelengths at higher concentrations of cystine indicating increased interaction between Ag NP surface atoms and cystine molecules. These observations confirm that cystine replaces the three types of surface coatings (citrate, PVP and $P E G)$. The higher shift at higher cystine concentrations indicates increased interaction between Ag NP surface atoms and cystine molecules.

The first peak shifts to higher wavelength with time; ca. $394-422 \mathrm{~nm}, 394-415 \mathrm{~nm}$, and 393-424 nm, for citrate-, PVP, and PEG coated Ag NPs six hours post interactions with cystine. The position of this peak remains constant beyond six hours up to 24 hours, indicating that cystine-Ag NP surface atom interaction reaches equilibrium within six hours.

The second peak is centered on $495 \mathrm{~nm}$ immediately after addition of cystine, shifts to higher wavelengths (ca. $530 \mathrm{~nm}$ ) between 0 and 6 hours and remains constant afterwards. These results indicate that cystine induces the aggregation of citrate, PEG and PVP coated Ag NPs, and that under the selected experimental conditions, Ag NPs occur both as primary Ag NPs as well as aggregated Ag NPs. These findings suggest that cystine binds to the surface of Ag NPs and replaces PVP and PEG coating similar to citrate, resulting in modification of NP surface coating and charge and therefore their aggregation behavior, in good agreement with other studies ${ }^{21}$. The Plasmon resonance peaks was stable within 6 hours indicating that Ag NPs were stable at this time point and did not undergo any further aggregation. No losses in the Plasmon resonance were observed at 24 hours compared to 6 hours indicating that aggregation resulted in the formation of small Ag NP aggregates that did not settle out of suspension. These findings are in good agreement with previous results suggesting that cystine induces aggregation of PVP-coated Ag NPs ${ }^{21,25}$.

\section{Environmental and Nanotoxicological Implications}


This research represents the first systematic study of the effect of cystine concentration on the aggregation of Ag NPs at ecotoxicologically relevant and near environmentally relevant Ag NP concentrations. Cystine significantly impacts the stability of citrate, PEG and PVP coated Ag NPs, resulting in Ag NP aggregation in a concentration-dependent manner, with a shift in the CCC toward lower concentrations of cystine at lower concentration of Ag NPs. SRFA reduced the cystine induced aggregation of Ag NPs within the limit of cystine solubility in water, likely due to the steric effect of SRFA. Consequently, in natural surface waters, it is likely that cystine will play an important role in determining the fate and behavior of cit-Ag NPs, and further research is required to investigate the interplay between cystine, electrolytes and NOM on the stability of $\mathrm{Ag} \mathrm{NPs}$. Due to the higher affinity of the cysteine thiol groups to $\mathrm{Ag}^{+}$and $\mathrm{Ag}$ surfaces compared with the cystine disulfide group, a similar concentration-dependent aggregation of $\mathrm{Ag}$ NPs in the presence of cysteine is expected. Several studies have demonstrated that cysteine induces aggregation of $\mathrm{Ag} \mathrm{NPs}^{25}$, but none has investigated the concentration-dependent aggregation of Ag NPs in the presence of cysteine.

In ecotoxicological studies of Ag NPs, cysteine is typically used to scavenge the dissolved ions and thus to differentiate dissolved vs. NP effects assuming no interaction occurs between Ag NPs and cysteine. Several papers have reported lower toxicity of Ag NPs in the presence of cysteine, and consequently concluded that silver ions are the major cause of their antimicrobial activity. Our results suggest that the reduced toxicity in the presence of cysteine widely observed in the literature could be due to a combined effect of $\mathrm{Ag}^{+}$scavenging by cysteine and Ag NP aggregation. The concentration-dependent aggregation of Ag NPs induced by cystine (a similar behavior is expected with cysteine) can alter the nature of the dose (toxicant) in a typical dose-response experiment; that is Ag NPs may form aggregates at low concentration and stay suspended as individual NPs at high Ag NP concentration.

This work underscores the importance of understanding the impact of biogeochemical molecules on NP stability in both ecotoxicological media as well as in the natural environment, and thus the fate and effects of NPs. This study also underscores the importance of investigating NP interactions at environmentally and toxicologically relevant NP concentrations.

\section{Acknowledgment}

We acknowledge funding from the National Science Foundation (NSF1437307), funding from the SmartState Center for Environmental Nanoscience and Risk (CENR), along with the Morris College and department of energy-environmental management (DOE-EM) summer research experience program. 
References

(1) NNI. National Nanotechnology Initiative. 2014.

(2) Royal Society \& Royal Academy Engineering Nanoscience and Nanotechnologies: Opportunities and Uncertainties. 2004.

(3) Mirshahghassemi, S.; Lead, J. R. Oil Recovery From Water Under Environmentally Relevant Conditions Using Magnetic Nanoparticles. Environ. Sci. Technol. 2015, 49 (19), 11729-11736.

(4) Woodrow Wilson data base. The project on emerging nanotechnologies (http://www.nanotechproject.org/). 2014.

(5) Benn, T. M.; Westerhoff, P. Nanoparticle Silver Released into Water From Commercially Available Sock Fabrics. Environ. Sci. Technol. 2008, 42 (11), 4133-4139.

(6) Kaegi, R.; Sinnet, B.; Zuleeg, S.; Hagendorfer, H.; Mueller, E.; Vonbank, R.; Boller, M.; Burkhardt, M. Release of Silver Nanoparticles From Outdoor Facades. Environ. Pollut. 2010, 158 (9), $2900-$ 2905.

(7) Sharma, V. K.; Siskova, K. M.; Zboril, R.; Gardea-Torresdey, J. L. Organic-Coated Silver Nanoparticles in Biological and Environmental Conditions: Fate, Stability and Toxicity. Adv. Colloid Interf. Sci. 2014, 204 (0), 15-34.

(8) Navarro, E.; Piccapietra, F.; Wagner, B.; Marconi, F.; Kaegi, R.; Odzak, N.; Sigg, L.; Behra, R. Toxicity of Silver Nanoparticles to Chlamydomonas Reinhardtii. Environ. Sci. Technol. 2008, 42 (23), 8959-8964.

(9) Smetana, A. B.; Klabunde, K. J.; Marchin, G. R.; Sorensen, C. M. Biocidal Activity of Nanocrystalline Silver Powders and Particles. Langmuir 2008, 24 (14), 7457-7464.

(10) Lubick, N. Nanosilver Toxicity: lons, Nanoparticles or Both? Environ. Sci. Technol. 2008, 42 (23), 8617.

(11) Miao, A.-J.; Luo, Z.; Chen, C.-S.; Chin, W.-C.; Santschi, P. H.; Quigg, A. Intracellular Uptake: A Possible Mechanism for Silver Engineered Nanoparticle Toxicity to a Freshwater Alga Ochromonas Danica. PLoS ONE 2011, 5 (12), e15196-1-e15196-8.

(12) Silva, T.; Pokhrel, L. R.; Dubey, B.; Tolaymat, T. M.; Maier, K. J.; Liu, X. Particle Size, Surface Charge and Concentration Dependent Ecotoxicity of Three Organo-Coated Silver Nanoparticles: Comparison Between General Linear Model-Predicted and Observed Toxicity. Sci. Tot. Environ. 2014, 468-469 (0), 968-976.

(13) El Badawy, A. M.; Silva, R. G.; Morris, B.; Scheckel, K. G.; Suidan, M. T.; Tolaymat, T. M. Surface Charge-Dependent Toxicity of Silver Nanoparticles. Environ. Sci. Technol. 2011, 45 (1), 283-287. 
(14) George, S.; Lin, S.; Ji, Z.; Thomas, C.; Li, L.; Mecklenburg, M.; Meng, H.; Wang, X.; Zhang, H.; Xia, T.; Hohman, J. N.; Lin, S.; Zink, J. I.; Weiss, P. S.; Nel, A. E. Surface Defects on Plate-Shaped Silver Nanoparticles Contribute to Its Hazard Potential in a Fish Gill Cell Line and Zebrafish Embryos. ACS Nano 2012, 6 (5), 3745-3759.

(15) Xiu, Z. M.; Zhang, Q. b.; Puppala, H. L.; Colvin, V. L.; Alvarez, P. J. J. Negligible Particle-Specific Antibacterial Activity of Silver Nanoparticles. Nano Lett. 2012, 12 (8), 4271-4275.

(16) Jin, X.; Li, M.; Wang, J.; Marambio-Jones, C.; Peng, F.; Huang, X.; Damoiseaux, R.; Hoek, E. M. V. High-Throughput Screening of Silver Nanoparticle Stability and Bacterial Inactivation in Aquatic Media: Influence of Specific lons. Environ. Sci. Technol. 2010, 44 (19), 7321-7328.

(17) Pokhrel, L. R.; Dubey, B.; Scheuerman, P. R. Natural Water Chemistry (Dissolved Organic Carbon, $\mathrm{PH}$, and Hardness) Modulates Colloidal Stability, Dissolution, and Antimicrobial Activity of Citrate Functionalized Silver Nanoparticles. Environ. Sci. Nano. 2014, 1 (1), 45-54.

(18) Kawata, K.; Osawa, M.; Okabe, S. In Vitro Toxicity of Silver Nanoparticles at Noncytotoxic Doses to HepG2 Human Hepatoma Cells. Environ. Sci. Technol. 2009, 43 (15), 6046-6051.

(19) Baalousha, M. Aggregation and Disaggregation of Iron Oxide Nanoparticles: Influence of Particle Concentration, PH and Natural Organic Matter. Sci. Tot. Environ. 2009, 407 (6), 2093-2101.

(20) Baalousha, M.; Sikder, M.; Prasad, A.; Lead, J.; Merrifield, R.; Chandler, G. T. The ConcentrationDependent Behavior of Nanoparticles. Env. Chem. (Online, http://dx. doi. org/10. 1071/EN15142) 2015.

(21) Yang, X.; Lin, S.; Wiesner, M. R. Influence of Natural Organic Matter on Transport and Retention of Polymer Coated Silver Nanoparticles in Porous Media. J. Hazard. Mater. 2014, 264 (0), 161168.

(22) Pokhrel, L. R.; Dubey, B.; Scheuerman, P. R. Impacts of Select Organic Ligands on the Colloidal Stability, Dissolution Dynamics, and Toxicity of Silver Nanoparticles. Environ. Sci. Technol. 2013, 47 (22), 12877-12885.

(23) Stewart, A.; Zheng, S.; McCourt, M. a. R.; Bell, S. E. J. Controlling Assembly of Mixed Thiol Monolayers on Silver Nanoparticles to Tune Their Surface Properties. ACS Nano 2012, 6 (5), 3718-3726.

(24) Mandal, S.; Gole, A.; Lala, N.; Gonnade, R.; Ganvir, V.; Sastry, M. Studies on the Reversible Aggregation of Cysteine-Capped Colloidal Silver Particles Interconnected Via Hydrogen Bonds. Langmuir 2001, 17 (20), 6262-6268.

(25) Gondikas, A. P.; Morris, A.; Reinsch, B. C.; Marinakos, S. M.; Lowry, G. V.; Hsu-Kim, H. CysteineInduced Modifications of Zero-Valent Silver Nanomaterials: Implications for Particle Surface Chemistry, Aggregation, Dissolution, and Silver Speciation. Environ. Sci. Technol. 2012, 46 (13), 7037-7045. 
(26) Römer, I.; Gavin, A. J.; White, T. A.; Merrifield, R. C.; Chipman, J. K.; Viant, M. R.; Lead, J. R. The Critical Importance of Defined Media Conditions in Daphnia Magna Nanotoxicity Studies. Toxicol. Lett. 2013, 0).

(27) Truong, L.; Zaikova, T.; Richman, E. K.; Hutchison, J. E.; Tanguay, R. L. Media Ionic Strength Impacts Embryonic Responses to Engineered Nanoparticle Exposure. Nanotoxicol. 2011, 6 (7), 691-699.

(28) Ciglenecki, I.; Cosovic, B.; Vojvodic, V.; Plavsic, M.; Furic, K.; Minacci, A.; Baldi, F. The Role of Reduced Sulfur Species in the Coalescence of Polysaccharides in the Adriatic Sea. Marine Chem. 2000, 71 (ЗГС̧ô4), 233-249.

(29) Van Vranken, D.; Weiss, G. Introduction to Bioorganic Chemistry and Chemical Biology; Garland Science: 2012.

(30) McBean, G. J. Astrocytes and the Regulation of Cerebral Cysteine/Cystine Redox Potential: Implications for Cysteine Neurotoxicity. In Cysteine: Biosynthesis, Chemical Structure and Toxicity; Chorkina, F. V., Karataev, A. I., Eds.; Nova Science Publishers: Hauppauge,NY, 2012.

(31) Banjac, A.; Perisic, T.; Sato, H.; Seiler, A.; Bannai, S.; Weiss, N.; K+!lle, P.; Tschoep, K.; Issels, R. D.; Daniel, P. T. The Cystine/Cysteine Cycle: a Redox Cycle Regulating Susceptibility Versus Resistance to Cell Death. Oncogene 2008, 27 (11), 1618-1628.

(32) Baalousha, M.; Nur, Y.; Römer, I.; Tejamaya, M.; Lead, J. R. Effect of Monovalent and Divalent Cations, Anions and Fulvic Acid on Aggregation of Citrate-Coated Silver Nanoparticles. Sci. Tot. Environ. 2013, 454-455 (0), 119-131.

(33) Römer, I.; White, T. A.; Baalousha, M.; Chipman, K.; Viant, M. R.; Lead, J. R. Aggregation and Dispersion of Silver Nanoparticles in Exposure Media for Aquatic Toxicity Tests. J. Chromatogr. A. 2011, 1218 (27), 4226-4233.

(34) Baalousha, M.; Lead, J. R. Rationalizing Nanomaterial Sizes Measured by Atomic Force Microscopy, Flow Field-Flow Fractionation, and Dynamic Light Scattering: Sample Preparation, Polydispersity, and Particle Structure. Environ. Sci. Technol. 2012, 46 (11), 6134-6142.

(35) Tejamaya, M.; Römer, I.; Merrifield, R. C.; Lead, J. R. Stability of Citrate, PVP, and PEG Coated Silver Nanoparticles in Ecotoxicology Media. Environ. Sci. Technol. 2012, 46 (13), 7011-7017.

(36) Chorover, J.; Zhang, J.; Amistadi, M. K.; Buffle, J. Comparison of Hematite Coagulation by Charge Screening and Phosphate Adsorption; Differences in Aggregate Structure. Clays and Clay Minerals 1997, 45 (5), 690-708.

(37) Domingos, R. F.; Peyrot, C.; Wilkinson, K. J. Aggregation of Titanium Dioxide Nanoparticles: Role of Calcium and Phosphate. Environ. Chem. 2010, 7 (1), 61-66.

(38) Baalousha, M.; Arkill, K. P.; Romer, I.; Palmer, R. E.; Lead, J. R. Transformations of Citrate and Tween Coated Silver Nanoparticles Reacted With Na2S. Sci. Tot. Environ. 2015, 502 (0), 344-353. 
(39) Moskovits, M.; Vlc ková, B. Adsorbate-Induced Silver Nanoparticle Aggregation Kinetics. J. Phys. Chem. B. 2005, 109 (31), 14755-14758.

(40) Elimelech, M.; O'Melia, C. R. Kinetics of Deposition of Colloidal Particles in Porous Media. Environ. Sci. Technol. 1990, 24 (10), 1528-1536.

(41) Malvern. Concentration limits for zeta potential measurements in the Zetasizer Nano. 2005.

(42) Lopez-Tobar, E.; Hernändez, B.; Ghomi, M.; Sanchez-Cortes, S. Stability of the Disulfide Bond in Cystine Adsorbed on Silver and Gold Nanoparticles As Evidenced by SERS Data. J. Phys. Chem. C. 2013, $117(3)$, 1531-1537.

(43) Podstawka, E.; Ozaki, Y.; Proniewicz, L. M. Adsorption of S-S Containing Proteins on a Colloidal Silver Surface Studied by Surface-Enhanced Raman Spectroscopy. Applied spectroscopy 2004, 58 (10), 1147-1156.

(44) Podstawka, E.; Ozaki, Y.; Proniewicz, L. M. Part I: Surface-Enhanced Raman Spectroscopy Investigation of Amino Acids and Their Homodipeptides Adsorbed on Colloidal Silver. Appl. Spectrosc. 2004, 58 (5), 570-580.

(45) Lee, H.; Kim, M. S.; Suh, S. W. Raman Spectroscopy of Sulphur-Containing Amino Acids and Their Derivatives Adsorbed on Silver. J. Raman Spectrosc. 1991, 22 (2), 91-96.

(46) Ravindran, A.; Dhas, S. P.; Chandrasekaran, N.; Mukherjee, A. Differential Interaction of Silver Nanoparticles With Cysteine. J. Exp. Nanosci. 2012, 8 (4), 589-595.

(47) Horovitz, O.; Tomoaia, G.; Mocanu, A.; Yupsanis, T.; Tomoaia-Cotisel, M. Protein Binding to Gold Colloids. Gold Bull 2007, 40 (3), 213-218.

(48) Brewer, S. H.; Glomm, W. R.; Johnson, M. C.; Knag, M. K.; Franzen, S. Probing BSA Binding to Citrate-Coated Gold Nanoparticles and Surfaces. Langmuir 2005, 21 (20), 9303-9307.

(49) Diegoli, S.; Manciulea, A. L.; Begum, S.; Jones, I. P.; Lead, J. R.; Preece, J. A. Interaction Between Manufactured Gold Nanoparticles and Naturally Occurring Organic Macromolecules. Sci. Tot. Environ. 2008, 402 (1), 51-61.

(50) Tipping, E.; Higgins, D. C. The Effect of Adsorbed Humic Substances on the Colloid Stability of Haematite Particles. Colloids Surf. 1982, 5 (2), 85-92.

(51) Baalousha, M.; Manciulea, A.; Cumberland, S.; Kendall, K.; Lead, J. R. Aggregation and Surface Properties of Iron Oxide Nanoparticles; Influence of PH and Natural Organic Matter. Environ. Toxicol. Chem. 2008, 27 (9), 1875-1882.

(52) Chen, K. L.; Elimelech, M. Influence of Humic Acid on the Aggregation Kinetics of Fullerene (C60) Nanoparticles in Monovalent and Divalent Electrolyte Solutions. J. Colloid Interf. Sci. 2007, 309 (1), 126-134. 

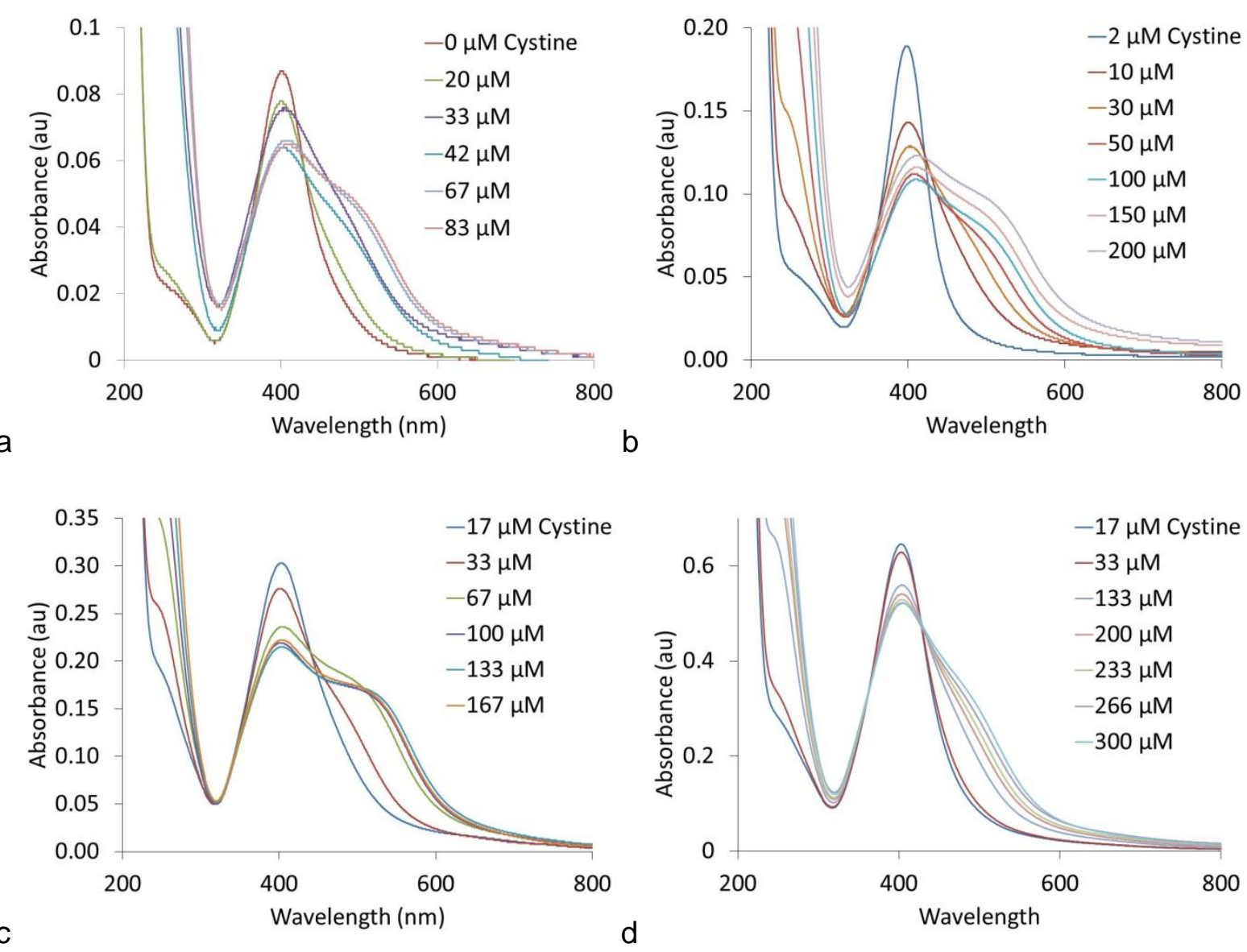

Figure 1. Uv-vis spectra of cit-Ag NP reacted with different concentrations of cystine at different concentrations of cit-AgNPs: (a) $125 \mu g \mathrm{~L}^{-1} \mathrm{Ag}$, (b) $250 \mu g \mathrm{~L}^{-1} \mathrm{Ag}$, (c) $500 \mu \mathrm{L} \mathrm{L}^{-1} \mathrm{Ag}$, and (d) $1000 \mu g \mathrm{~L}^{-1} \mathrm{Ag}$ 
a
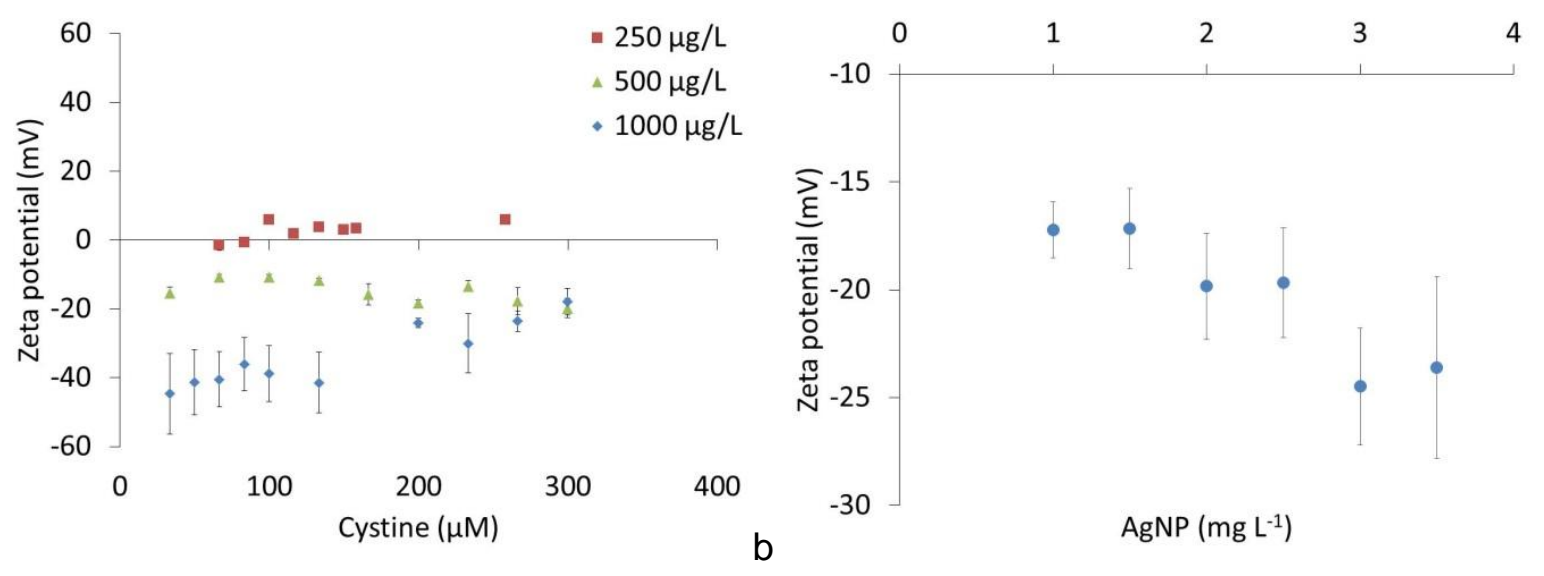

b

$\operatorname{AgNP}\left(\mathrm{mg} \mathrm{L}^{-1}\right)$

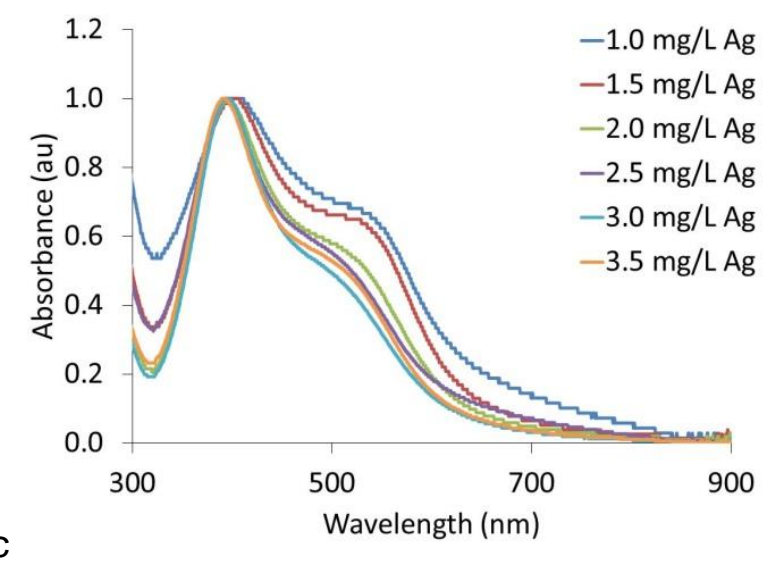

Figure 2. Zeta potential of cit-Ag NPs reacted with cystine (a) at different concentrations of cystine and cit-Ag NPs, and (b) at fixed concentration of cystine (225 $\mu \mathrm{M})$ and different concentrations of cit-Ag NPs (1-3.5 mg L-1). (c) Uv-vis spectra of cit-Ag NPs 24 hours post interaction with $225 \mu \mathrm{M}$ cysteine. 
a
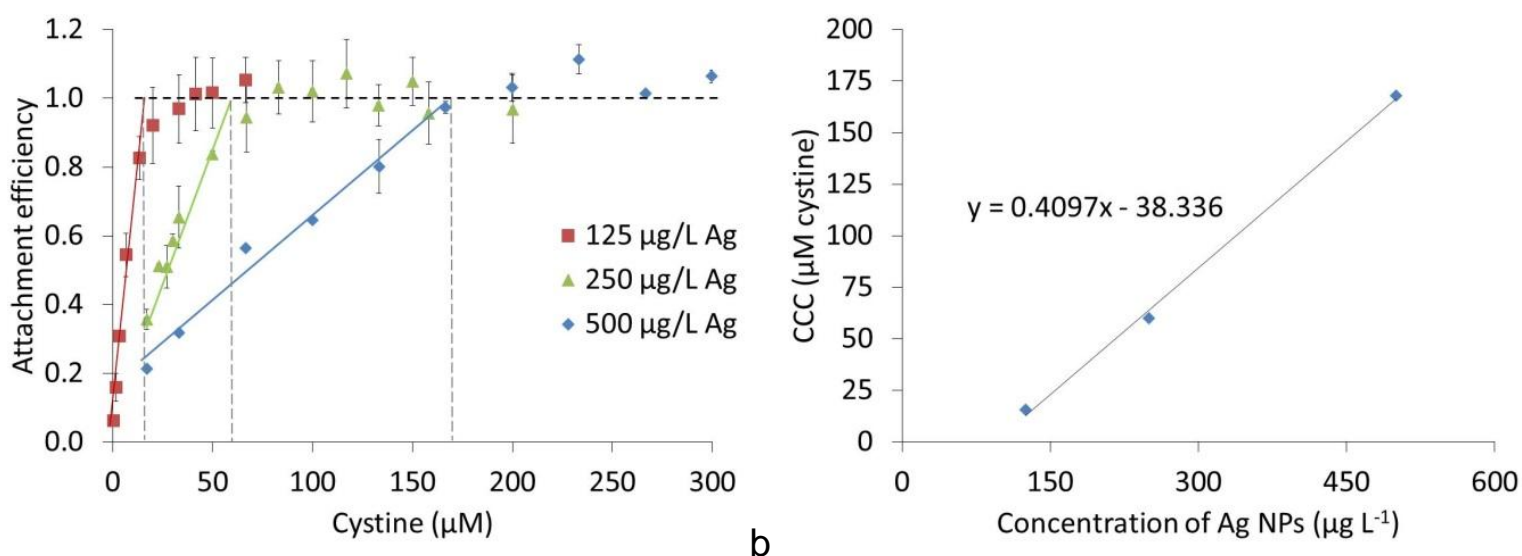

Figure 3. Attachment efficiency of cit-Ag NPs reacted with cystine at different concentrations of cit-Ag NPs. 

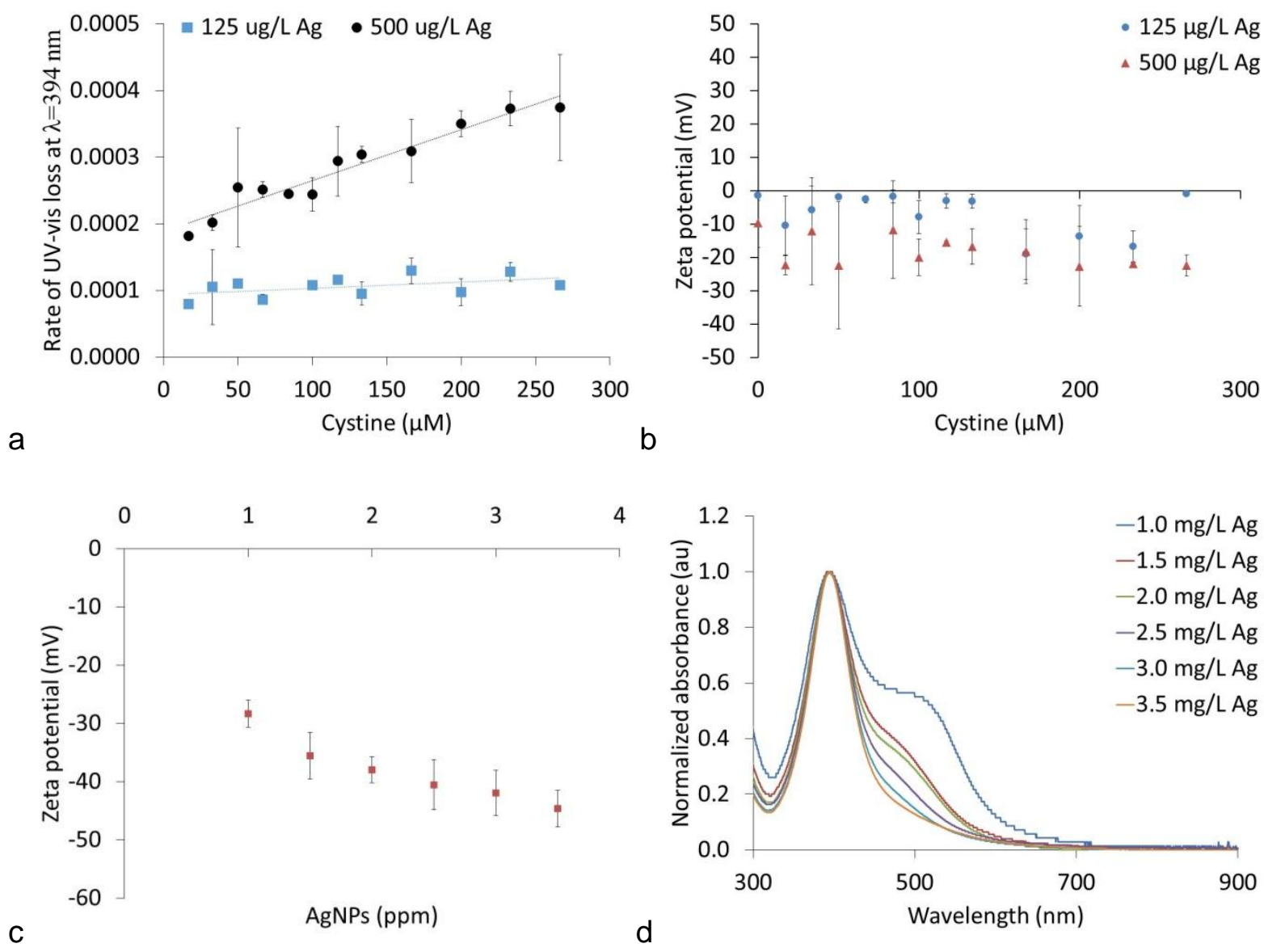

Figure 4. (a) rate loss of the UV-vis absorbance at $394 \mathrm{~nm}$ as a function of cystine concentration in the presence of $1 \mathrm{mgL}^{-1}$ SRFA, (b) zeta potential of cit-Ag NPs reacted with cystine in the presence of $1 \mathrm{mgL}^{-1}$ SRFA, and (c) zeta potential of cit-Ag NPs reacted with fixed concentration of cystine $(225 \mu \mathrm{M})$ and different concentrations of cit-Ag NPs (1-3.5 mg L $\left.\mathrm{L}^{-1}\right)$. (d) normalized Uv-vis spectra of cit-AgNPs 24 post reaction with cystine. 


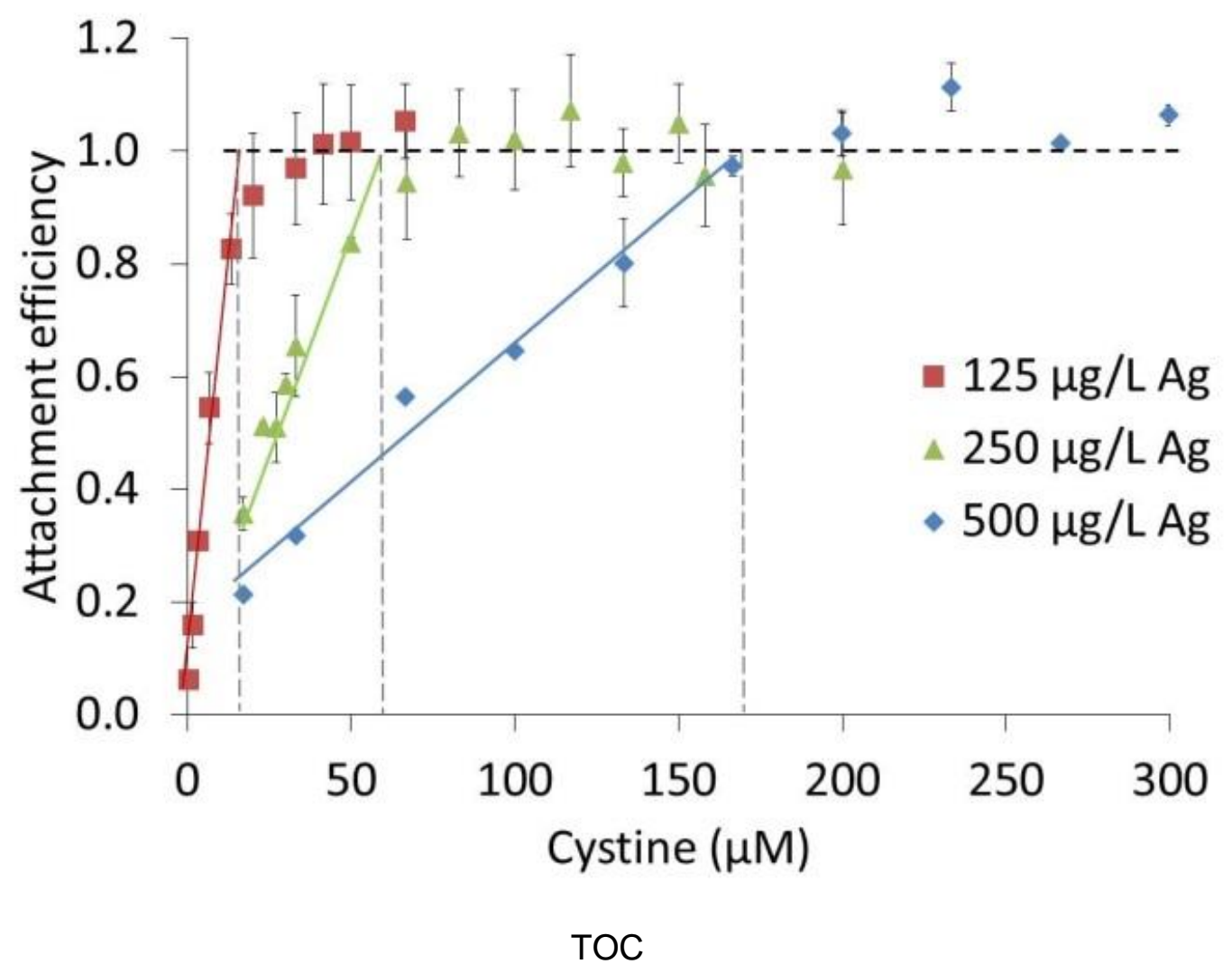

